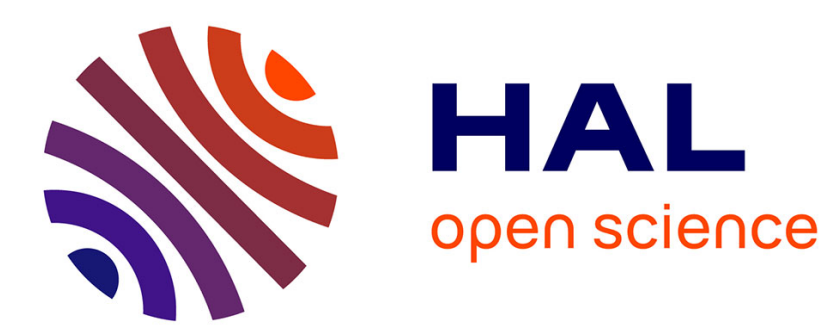

\title{
Cellular correlates of wakefulness and slow-wave sleep: evidence for a key role of inhibition
}

\author{
Eduarda Susin, Alain Destexhe
}

\section{To cite this version:}

Eduarda Susin, Alain Destexhe. Cellular correlates of wakefulness and slow-wave sleep: evidence for a key role of inhibition. Current Opinion in Physiology, 2020, 15, pp.68-73. 10.1016/j.cophys.2019.12.006 . hal-02418976

\section{HAL Id: hal-02418976 https://hal.science/hal-02418976}

Submitted on 19 Dec 2019

HAL is a multi-disciplinary open access archive for the deposit and dissemination of scientific research documents, whether they are published or not. The documents may come from teaching and research institutions in France or abroad, or from public or private research centers.
L'archive ouverte pluridisciplinaire HAL, est destinée au dépôt et à la diffusion de documents scientifiques de niveau recherche, publiés ou non, émanant des établissements d'enseignement et de recherche français ou étrangers, des laboratoires publics ou privés. 
Current Opinion in Physiology, in press

\title{
Cellular correlates of wakefulness and slow-wave sleep: evidence for a key role of inhibition ${ }^{\star}$
}

\author{
Eduarda Susin ${ }^{\mathrm{a}}$, Alain Destexhe $\mathrm{a}^{\mathrm{a}, *}$ \\ ${ }^{a}$ Department of Integrative and Computational Neuroscience (ICN), Paris-Saclay Institute of Neuroscience (NeuroPSI), \\ Centre National de la Recherche Scientifique (CNRS), 91198 Gif-sur-Yvette, France
}

\begin{abstract}
Recent studies have identified interesting cellular dynamics in wakefulness and slow-wave sleep (SWS), as we review here for unit recordings in animals and human. First, a novel form of homeostasis was observed during sleep, where high-firing cells in wake tend to fire slower during SWS, while low-firing cells in wake tend to fire faster in SWS. Second, there seems to be a stronger involvement of inhibitory cells compared to excitatory cells during gamma oscillations in SWS. Third, pairwise cross-correlations between cells seem to increase specifically during SWS, but only for inhibitory neurons. We compare these results between animal and human unit recordings, and confirm the similarities in cellular dynamics in wake and SWS, and highlight that differences are mostly apparent in the dynamics and correlations of inhibitory cells. These results suggest that inhibitory networks are key in the dynamics of sleep, and should be a main target in future studies.
\end{abstract}

Keywords: Wakefulness, Slow-wave sleep, Unit activity, Gamma oscillations, Utah-array, Human, Intracranial recordings

2010 MSC: 00-01, 99-00

\section{Introduction}

Slow-wave sleep (SWS) is characterized by the prevalence of slow waves in the delta $(0.5-4 \mathrm{~Hz})$ frequency range, which are visible in the electroencephalogram (EEG) and local field potentials (LFPs). Early studies using intracellular recordings in anesthetized sleeping animals [1, 2, 3, have shown that the depth-positive

\footnotetext{
${ }^{\star}$ Research supported by Centre National de la Recherche Scientifique (CNRS), the European Community (Human Brain Project, H2020-785907), and École des Neurosciences de Paris (ENP).

${ }^{*}$ Corresponding author

Email addresses: eduardadsusin@gmail.com (Eduarda Susin), destexhe@unic.cnrs-gif.fr (Alain Destexhe) 
5 (surface-negative) EEG components of slow waves are associated with cellular hyperpolarization and pause of firing. In natural SWS, extracellularly-recorded neurons fire in coincidence with the depth-negative component of slow-wave complexes, whereas the depth-positive component is associated with neuronal silence [4, 5, 6]. Similar relations were found for delta waves in the intact brain [7], or isolated cortex [8]. Thus it seems that slow-wave complexes are characterized by alternating periods of sustained firing and neuronal silence usually called "Up" and "Down" states, respectively. It was shown that, in natural sleep, Up and Down states appear synchronously in multiple cells recorded extracellularly [6, and the relation between the slow-wave and cellular hyperpolarization was later confirmed by intracellular recordings of neurons in naturally sleeping animals [9]. Note that Down states are not periods of complete silence. A possible relation between the residual activity during Down states and memory consolidation has been proposed based on extracellular recordings in prefrontal cortical areas in rats 10. This reminiscent activity during down states was also observed in human recordings 11.

Early studies also showed that, in aroused states, cortical cells fire tonically [12, 13, 14, and the EEG is dominated by low-amplitude fast activity in the beta/gamma (15-75 Hz) frequency range. Extracellular studies in natural SWS found that all characteristics from the spiking activity, LFP activity or LFP coherence, are identical between the Up-states of SWS, wakefulness or rapid-eye movement (REM) sleep 6]. These seemingly identical properties led to the suggestion that SWS Up-states constitute small episodes of activity replayed from wakefulness, possibly involved in memory consolidation [15. This view was challenged recently by studies who found differences in firing activity between natural SWS and wakefulness [16. 17, 18, 19, 20], as we review in this paper.

\section{Cellular correlates during wakefulness and slow-wave sleep}

Generic features of brain activity during wake and sleep states in humans are illustrated in Figure 1 . We used a dataset where units could be separated into fast-spiking (FS), presumed inhibitory, and regularspiking (RS), presumed excitatory cells; some of these were confirmed by direct functional identification [19]. In these human recordings, during Wake and REM states, the activity of both RS and FS cells is sustained, asynchronous and irregular, and the LFP is dominated by high-frequency activity. In contrast, during SWS, the activity is dominated by slow waves in the LFP, which are paralleled with synchronized silences (Down states) where almost all cells cease firing. Outside these periods (Up states), the dynamics is sustained, asynchronous and irregular, similar to Wake and REM states. These observations lead to the statement that the Down states is what makes SWS dynamics more synchronized, and in a sense, the higher synchrony during SWS does not come from the firing of units, but rather comes from these periods of non-firing.

Consistent with this interpretation, a previous extracellular study in marmoset cortical areas [18], found that there was no significant increase of neural correlation during SWS when recording spontaneous activity, 
providing another evidence that SWS Up-states display firing dynamics almost identical to wakefulness.

However, several observations have shown differences in spiking activity during wake states and SWS. 40 For instance, a relevant feature that has been taken into consideration is the firing level of single units in different states. A recent work using wide-field calcium imaging in cortical superficial and deep layers in mice [17] showed that the activity of excitatory neurons was highest during Wake, followed by that of SWS and subsequently followed by that of REM sleep (Wake > SWS > REM). Using two-photon imaging in layer $2 / 3$, the same study [17] also showed that paralvabumin-positive (PV+) interneuron activity follow the relation: Wake $=$ REM $>$ SWS, while somatostatin-positive interneurons follow: Wake $>$ SWS $>$ REM. In human recordings, the firing rates were comparable in all brain states [21], which would tend to support that the recorded FS cells are PV+ cells, although this should be confirmed with better statistics.

In addition, a recent analysis [16] found important differences between SWS and Wake by focusing on Up states. By means of silicon probes implanted in frontal cortical areas of rats, measurements of extracellular signals allowed simultaneous identification of brain state and the assessment of the firing pattern of putative excitatory and inhibitory cells. This analysis [16] showed that during SWS pyramidal cells that displayed high-firing during Wake tended to decrease their activity during SWS, while cells that displayed low-firing during wake tended to increase their firing during SWS. We confirm these findings also in humans through similar recordings and data processing performed previously [19]. Figure 2 compares the results from [16] with our own analysis based on the data coming from [19. Despite the small number of cells available in the human data set, in a confidence interval of $95 \%$, it is possible to observe a small slope with respect to the identity (0.67-0.94). These results (in both data sets) indicate that a new type of homeostasis, as argued by [16, in which a homogenization of firing is observed thorough the differential action in cells with different levels of activity (in opposition to previous homeostatic models [22, 23]). On the other hand, when considering putative inhibitory cells the result is divergent in the two data sets (rat and human). No firing alteration is observed in the firing of inhibitory cells during Wake and SWS in rat [16, while a tendency close to that which is observed with excitatory cells is observed in human inhibitory cells. This result should be checked in data sets where more neurons are available.

A further difference observed during Up states in SWS with respect to Wake states is the involvement of inhibitory cells in gamma oscillations. As recently shown in human recordings [11, both putative excitatory regular-spiking and putative inhibitory fast-spiking populations increase their firing during gamma oscillations. Here, by analyzing the pre-processed data from [19], we noted that there is a significant increase on the average firing of the inhibitory population during SWS with respect to its activity during Wake (see Fig. 3D). While no significant increase in firing with respect to Wake is exhibited by the excitatory population during SWS (see Fig. 3E). Indicating an increase of FS participation in gamma generation during SWS.

Another key aspect to differentiate the network state between Wake and SWS is the pairwise correlations displayed by unit spikes. Figure 4 A shows recordings of multielectrode arrays implanted in two monkey 
cortical areas (premotor dorsal - PMd - and motor neocortical -MI, layers II/III) [11, in which spiking activity and LFP were simultaneously recorded. Cross-correlations between spike trains were normalized by the geometric mean of each cell pair's average firing rates to avoid spurious effects from cell-intrinsic firing levels 24. Remarkably, comparing the correlation matrices between Wake and SWS, one sees that pairs of cells become correlated specifically during SWS (Fig. 4). These sleep-specific correlations appear even between distant cortical areas. Interestingly, these pairwise spike train correlations only concern pairs of FS cells. With this observation we emphasize one more time that there seems to exist a particularly high involvement of inhibitory cells specifically during SWS. In agreement, a previous study [18 also reported an increase of correlations during SWS in the response to auditory stimuli, but no separation between RS and FS cells was attempted in that study.

Furthermore, this particular involvement of inhibition can also be detected from correlated patterns analyzed using maximum entropy models. Such methods can infer correlations from large data sets with the advantage that they can uncover collective dynamics with very few ad hoc assumptions. Recently, spiking activity in human recordings were used to infer the parameters of a Maximum Entropy model that takes into account only and exactly the single neurons' spiking probability, and the pairwise covariances observed in the data 20. In this work, illustrated in Fig. 4 $4 \mathrm{~B}$, the empirical probability distributions of population activity of FS and RS cells, during Wake and SWS, were compared to the probability distributions predicted by the model. Population activity was defined as the average number of active cells in a certain time window. This work showed that a model based exclusively on pairwise couplings can successfully predict the activity of both neuron types during Wake states (see the measures of the Kullback-Leibler divergence, $D_{K L}$, in Fig. 4p1). However, it also demonstrated that pairwise interactions alone are not enough to describe the probability distribution displayed by the FS population during SWS (Fig. 4 4 2). This result implies that, in contrast to the RS population, higher-order, or even population-wide, interactions govern the behavior of FS cells during SWS. This indicates another important difference between Wake and SWS states with respect to FS cells.

\section{Discussion}

In this paper, we briefly reviewed a few of recent studies about the cellular correlates of wake and sleep states based on multiple unit recordings. Similar to a previous study in cats [6], it was found that in human and monkey multielectrode recordings, wake and sleep states globally display similar levels of firing. Most units fire irregularly and asynchronously, and this pattern is seen in all brain states (Fig. 1). Note that, in mice, a different level of activity is observed between Wake and sleep depending on the cell type. In the light of recent studies [11, 16, 17, 20, we also found differences between Wake and SWS in human: (1) there seems to exist a new type of homeostasis of firing activity, where high-firing cells in wake tend to fire lower 
during SWS, and vice-versa for low-firing cells, as found in rats [16] and which we confirm here in human (Fig. 2) for RS and FS cells. (2) There is a stronger participation of FS cells during gamma oscillations in SWS compared to Wake (Fig. 3). (3) Besides the level of firing, there is also an increase of the spiking correlation between pairs of FS cells, specifically during sleep [11] (Fig. 4).

Taken together, these results show that inhibition seems to be a key to differentiate the network state between Wake and SWS. This is true for the the level of firing of inhibitory cells, which seems to be maximally involved during SWS gamma oscillations. It is also true for the level of correlation between inhibitory cells, which show remarkably high correlations that specifically appear during SWS, sometimes extending to large cortical distances [11] (Fig. 44A).

We presently do not know why inhibitory networks seem different during sleep. A possibility is that the different levels of cholinergic drive in Wake and SWS may up-regulate some of the inhibitory neurons, resulting in an increase of participation in gamma oscillations, as we observed. However, this does not explain why inhibitory cells are so correlated during SWS, which necessarily comes from an excitatory drive yet to be identified.

\section{Acknowledgments}

This research was supported by the Centre National de la Recherche Scientifique (CNRS) and the European Community (Human Brain Project, H2020-785907). E.S. acknowledges a PhD fellowship from the École des Neurosciences de Paris (ENP).

\section{References}

[1] M. Steriade, A. Nunez, F. Amzica, Intracellular analysis of relations between the slow $(<1 \mathrm{hz})$ neocortical oscillation and other sleep rhythms of the electroencephalogram, Journal of Neuroscience 13 (8) (1993) 3266-3283 (1993).

[2] M. Steriade, D. Contreras, R. C. Dossi, A. Nunez, The slow ( $<1 \mathrm{hz}$ ) oscillation in reticular thalamic and thalamocortical neurons: scenario of sleep rhythm generation in interacting thalamic and neocortical networks, Journal of Neuroscience 13 (8) (1993) 3284-3299 (1993).

[3] D. Contreras, M. Steriade, Cellular basis of eeg slow rhythms: a study of dynamic corticothalamic relationships, Journal of Neuroscience 15 (1) (1995) 604-622 (1995).

[4] M. Steriade, D. Contreras, F. Amzica, I. Timofeev, Synchronization of fast (30-40 hz) spontaneous oscillations in intrathalamic and thalamocortical networks, Journal of Neuroscience 16 (8) (1996) 2788-2808 (1996).

[5] F. Amzica, M. Steriade, The k-complex: its slow (<1-hz) rhythmicity and relation to delta waves, Neurology 49 (4) (1997) 952-959 (1997).

[6] A. Destexhe, D. Contreras, M. Steriade, Spatiotemporal analysis of local field potentials and unit discharges in cat cerebral cortex during natural wake and sleep states, Journal of Neuroscience 19 (11) (1999) 4595-4608 (1999).

[7] G. Buzsaki, R. G. Bickford, G. Ponomareff, L. Thal, R. Mandel, F. H. Gage, Nucleus basalis and thalamic control of neocortical activity in the freely moving rat, Journal of neuroscience 8 (11) (1988) 4007-4026 (1988).

[8] J. D. Frost Jr, P. Kellaway, A. Gol, Single-unit discharges in isolated cerebral cortex, Experimental neurology 14 (3) (1966) 305-316 (1966). 
[9] M. Steriade, I. Timofeev, F. Grenier, Natural waking and sleep states: a view from inside neocortical neurons, Journal of neurophysiology 85 (5) (2001) 1969-1985 (2001).

[10] R. Todorova, M. Zugaro, Isolated cortical computations during delta waves support memory consolidation, Science 366 (6463) (2019) 377-381 (2019).

[11] M. Le Van Quyen, L. E. Muller, B. Telenczuk, E. Halgren, S. Cash, N. G. Hatsopoulos, N. Dehghani, A. Destexhe, Highfrequency oscillations in human and monkey neocortex during the wake-sleep cycle, Proceedings of the National Academy of Sciences 113 (33) (2016) 9363-9368 (2016).

[12] D. H. Hubel, Single unit activity in striate cortex of unrestrained cats, The Journal of physiology 147 (2) (1959) 226-238 (1959).

[13] E. V. Evarts, Temporal patterns of discharge of pyramidal tract neurons during sleep and waking in the monkey, Journal of neurophysiology 27 (2) (1964) 152-171 (1964).

[14] M. Steriade, M. Deschenes, Inhibitory processes and interneuronal apparatus in motor cortex during sleep and waking. ii. recurrent and afferent inhibition of pyramidal tract neurons., Journal of Neurophysiology 37 (5) (1974) $1093-1113$ (1974).

[20] T.-A. Nghiem, B. Telenczuk, O. Marre, A. Destexhe, U. Ferrari, Maximum-entropy models reveal the excitatory and inhibitory correlation structures in cortical neuronal activity, Physical Review E 98 (1) (2018) 012402 (2018).

[21] N. Dehghani, A. Peyrache, B. Telenczuk, M. Le Van Quyen, E. Halgren, S. S. Cash, N. G. Hatsopoulos, A. Destexhe, Dynamic balance of excitation and inhibition in human and monkey neocortex, Scientific reports 6 (2016) 23176 (2016).

[22] G. Tononi, C. Cirelli, Sleep and the price of plasticity: from synaptic and cellular homeostasis to memory consolidation and integration, Neuron 81 (1) (2014) 12-34 (2014).

[23] G. G. Turrigiano, S. B. Nelson, Homeostatic plasticity in the developing nervous system, Nature reviews neuroscience 5 (2) (2004) 97 (2004).

[24] J. De La Rocha, B. Doiron, E. Shea-Brown, K. Josić, A. Reyes, Correlation between neural spike trains increases with firing rate, Nature 448 (7155) (2007) 802 (2007).

\section{Highlighted references}

- Ref. 9]: first paper having shown the dynamics of slow-wave sleep intracellularly in cats, where the Down-states are clearly associated with cellular hyperpolarization, while Up-states are intracellularly very close to the dynamics found during wakefulness.

- Ref. [16]: this paper showed clear evidence for a regulation of the firing of neurons between Wake and SWS states, where neurons with high levels of firing tend to fire lower during SWS, and conversely, 
neurons with low levels of firing tend to fire higher during SWS. This can be interpreted as a form of homeostasis of firing dynamics between wake and sleep states.

- Ref. 17]: this paper showed features of the neural activity of wake and sleep states in mice. This was done for excitatory cells, which fired more during Wake than the two sleep states, while interneurons also tended to fire more, but with some differences according to the type of inhibitory cell.

- Ref. 20]: this study confirmed that inhibitory neurons follow a very particular dynamics during sleep, pairwise correlations cannot account for their firing statistics, and higher-order correlations are needed.

- Ref. [1]: this paper investigated the firing of excitatory and inhibitory cells in human and monkey during wake and sleep states, during gamma oscillations. A higher level of participation was found for inhibitory cells, as well as high correlations, specific to SWS, and only between inhibitory cells. 

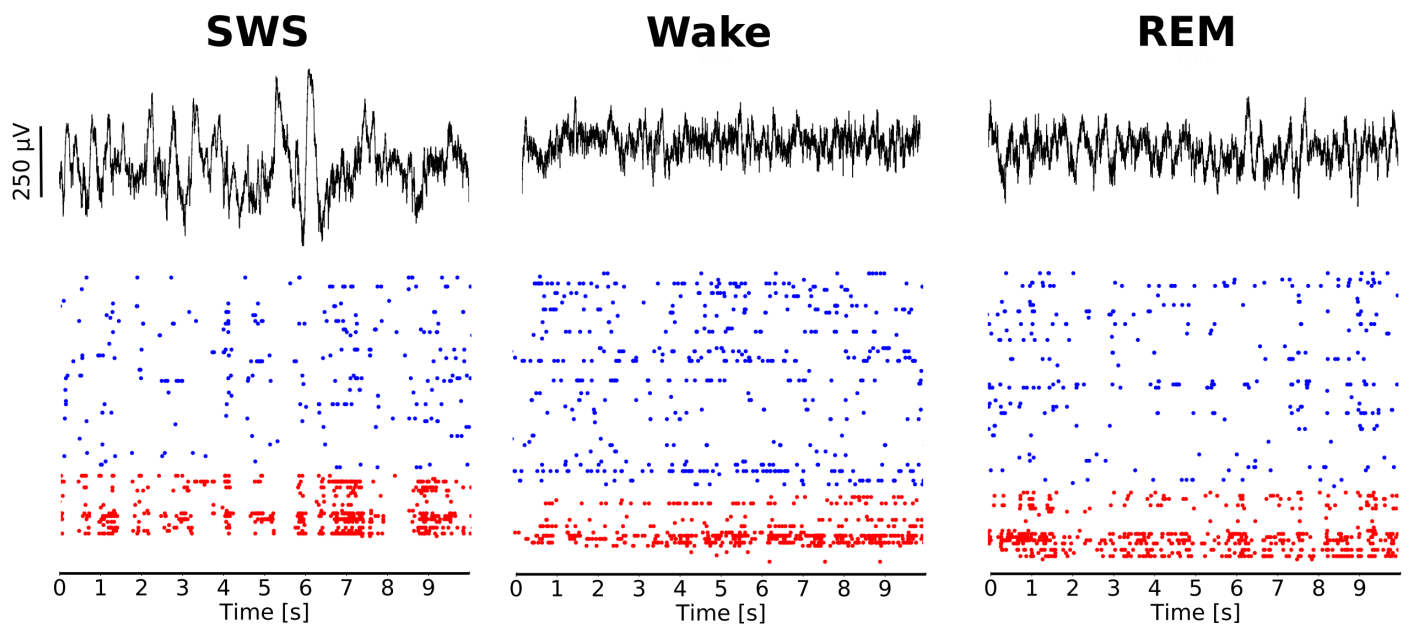

Figure 1: Features of different brain states in Human. Selected parts of 10 seconds of recordings of LFP (top) and spiking activity (bottom) of SWS, Wake and REM are shown. The data were recorded from the same human subject which had a multielectrode array implanted in temporal cortex before a therapeutic surgery. LFP and spiking activity were simultaneously measured and the spikes were sorted allowing the identification of 68 putative excitatory (blue) and 23 putative inhibitory (red). Data sample from 19. 
A

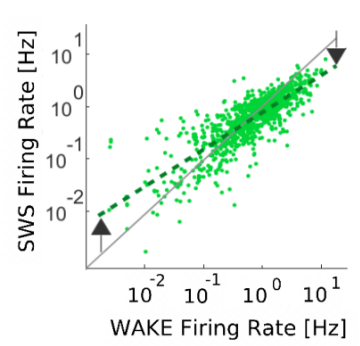

\section{Rat}

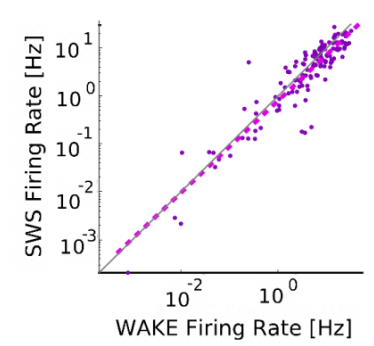

B

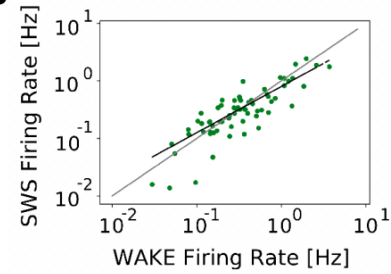

Human

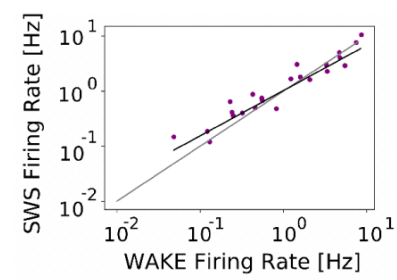

Figure 2: Comparison of firing rate during Wake and SWS states in rat and human cortex. Each point in the graph characterizes the firing rate (log scale) of an individual cell in the two states. Putative excitatory cells, both in rat and humans, suffer a change in their firing rate depending of their level of activity. Neurons that are highly active during wake decrease their firing during SWS, while neurons that have very low firing rates increase their firing (see arrows). Diverging results were found in the two data sets when looking to putative inhibitory cells. A) Putative excitatory and inhibitory neurons in rat (slopes, 95\% confidence interval: $0.66-0.71$ and 0.996-1.005). Figure adapted with permission from [16]. B) Putative excitatory and inhibitory neurons in human (slopes, 95\% confidence interval: 0.67-0.94 and 0.68-0.94). Data sample from [19]. 

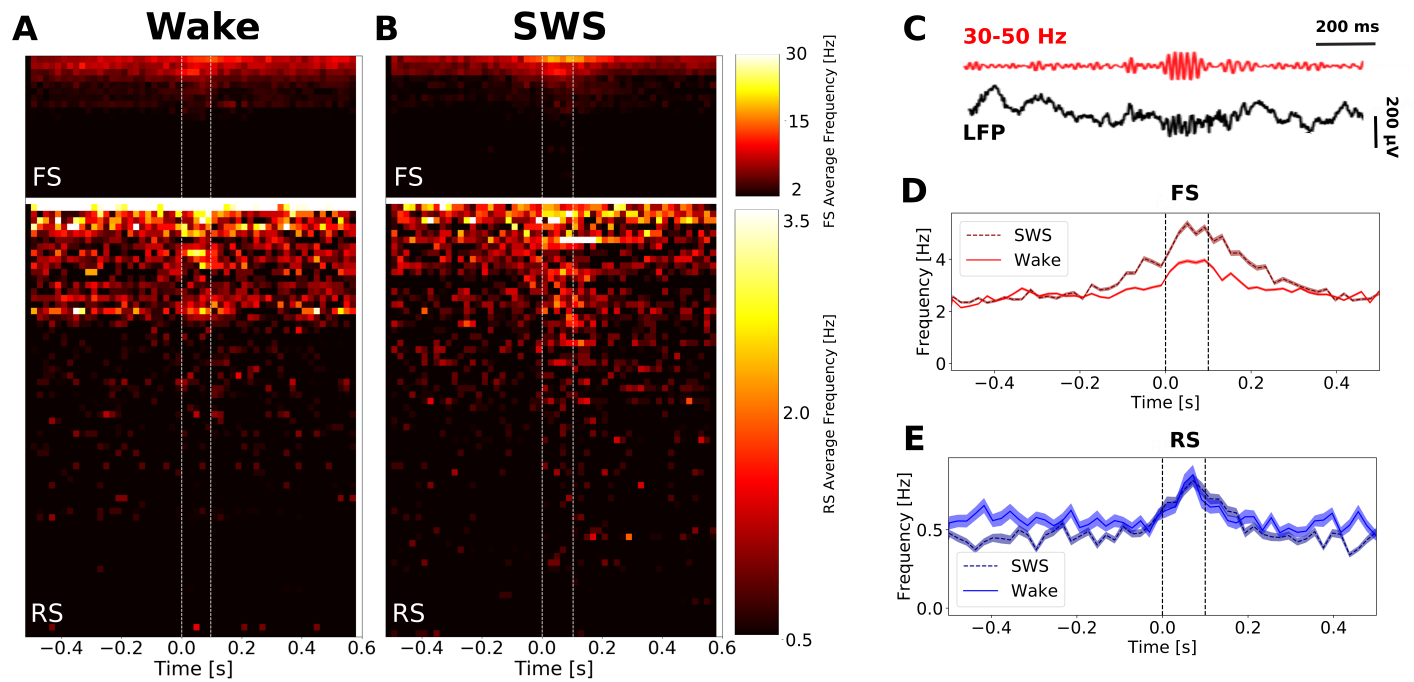

Figure 3: Difference of firing rates during $\gamma$ events in RS and FS cells in Human Wake and SWS states. The color-maps indicating the average firing rate per cell around $\gamma$ events in both Wake and SWS states are indicated in A and B. The firing of regular spiking (RS) are displayed in the bottom and the fast spiking (FS) cells is displayed in the top. Each line indicates the color-coded firing rate of each neuron in time averaged between all the gamma periods detected. For each $\gamma$ event the firing rate was calculated in bins of $20 \mathrm{~ms}$ around the centered $\gamma$ period. The white dashed lines indicate the beginning and the average end of $\gamma$ periods identified in the data. Gamma periods were identified by the variation of the envelope obtained through the Hilbert Transform of the filtered LFP in the range of 30-50 Hz as is schematically shown in C. Neurons were ordered by their discharge probability during $\gamma$. The average firing rate per population during $\gamma$ events in Wake and SWS are displayed in D and E. The color-coded firing rates shown in A and B were average in between neurons for each population: FS (D) and RS (E). The shadowed parts indicate the standard error of the mean. Data sample from [19]. 
A
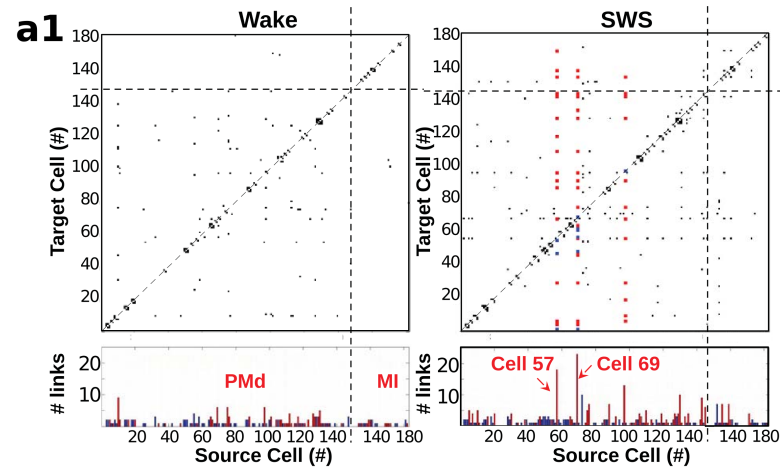

a2

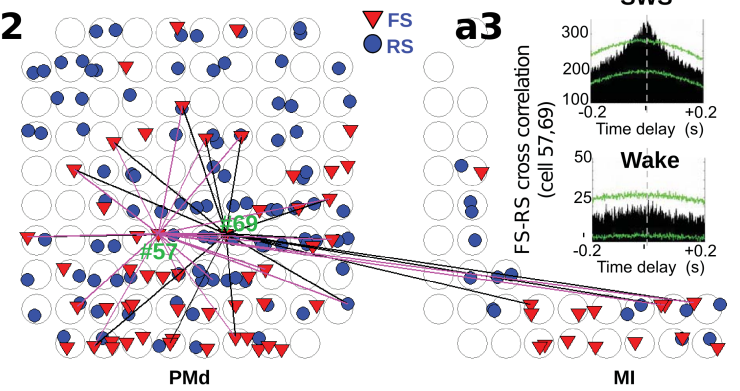

B

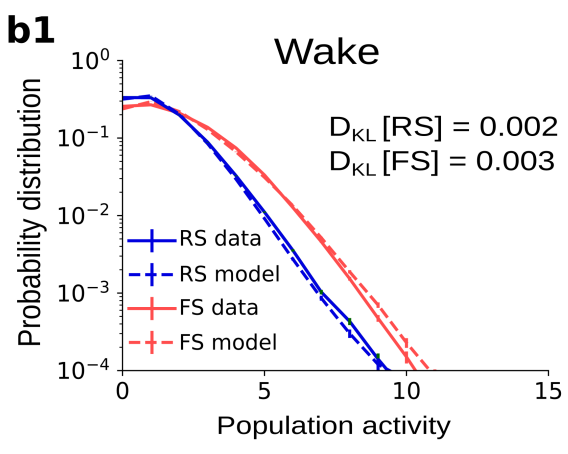

b2

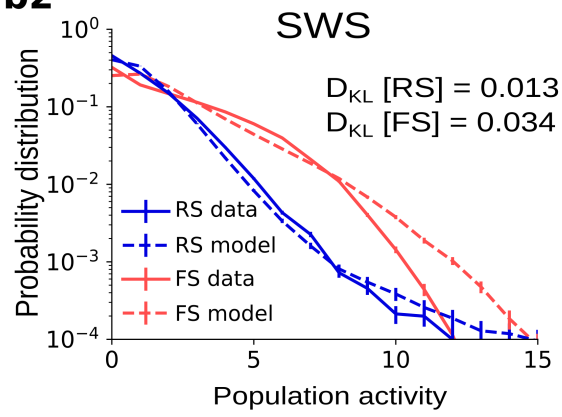

Figure 4: Difference in pairwise spike train cross-correlations among RS and FS cells during Wake and SWS. A) Spiking activity measured during $\beta$ oscillations through multielectrod arrays in two cortical areas in monkey (premotor dorsal, $\mathrm{PMd}$, and motor neocortical, MI). Neurons could be identified as RS and FS. a1) Cell-cell correlations matrices containing cells from the two areas (PMd and MI). For cells 57/69 (during SWS) the type of target cells is indicated with colors (red, FS; and blue, RS). a2) Spatial location of different cells in the implanted arrays. Note that FS cells (exemplified by cells 57 and 69 ) display synchronous activity even with cell recorded in another cortical area (M1). a3) Spike cross-correlogram between cells 57 and 69 during SWS and Wake states. A significant peak is observed only during SWS. Figure A was adapted from [11. B) Empirical probability distributions of human spiking activity are compared with the ones predicted by Maximum Entropy model (see main text), both in Wake (b1) and SWS (b2) for two type of cells (FS and RS). Measures of the Kullback-Leibler divergence, $D_{K L}$, are indicated in the graphs. Figure B was adapted from 20. 